\title{
Assessing the potential of ubiquitous computing for improving business process performance
}

\author{
Nina Oertel $\cdot$ Jens Dibbern $\cdot$ Zoltan Nochta
}

Received: 21 January 2009/Revised: 27 August 2009/Accepted: 17 September 2009/

Published online: 24 November 2009

(C) Springer-Verlag 2009

\begin{abstract}
Although ubiquitous technologies such as RFID, sensor networks, and networked embedded systems are quite mature, widespread adoption by organizations has yet to take place. This may be due to the lack of systematic assessment of the potential of ubiquitous technologies for creating value. Accordingly, a prescriptive model is presented that shows how value is created through the fit between generic capabilities of ubiquitous technologies and task characteristics in business processes. The identified task characteristics can thus be used as indicators for assessing the potential improvements in business process performance through particular ubiquitous computing functionalities.
\end{abstract}

Keywords Ubiquitous technologies (UT) · Information system (IS) · Business information System (BIS) - Radio frequency identification (RFID) · Task-technology fit (TTF)

\section{Introduction}

As ever cheaper, smaller and more powerful hardware components are developed, it becomes feasible to embed miniature computers into real-world objects, resulting in "smart items" (Mattern 2005). Smart items consist of a physical component (the object) and an information processing component. Current technologies employed

N. Oertel $\cdot$ Z. Nochta

SAP Research, Vincenc-Priessnitz-Straße 1, 76131 Karlsruhe, Germany

e-mail: nina.oertel@sap.com

Z. Nochta

e-mail: zoltan.nochta@sap.com

J. Dibbern $(\bowtie)$

Institute of Information Systems, University of Bern, Engehaldenstrasse 8, 3012 Bern, Switzerland e-mail: jens.dibbern@iwi.unibe.ch 
to enhance real world objects include radio frequency identification (RFID) systems, sensor networks, and networked embedded systems. Enhancing the abilities of human beings with mobile computing devices is another way of embedding information technology in the real world. In conjunction, these technologies contribute to realizing the vision of ubiquitous computing that was defined by Weiser (1993) as "the method of enhancing computer use by making many computers available throughout the physical environment, but making them effectively invisible to the user". The terms ubiquitous and pervasive computing are often used synonymously (Satyanarayanan 2002), although Lyytinen and Yoo (2002) claim that pervasive computing is characterized by a lower level of mobility than ubiquitous computing. As a seamless user interface is of lower importance in a business context, ubiquitous computing will in the following be understood as populating the world with smart items. Technologies suited for this purpose are called ubiquitous technologies (UT).

Among the benefits that have been attributed to UT in the context of business applications are the improved coordination in supply chains (Lee and Özer 2007), increased visibility and responsiveness (Wamba and Ygal 2008), as well as improved safety and security of supply chains (Inaba 2008). However, the benefits are opposed by the costs for UT, the costs for the necessary infrastructure as well as the risks associated with embedding UT in the business context. Most notably, it has been argued that UT puts the privacy of consumers at risk (Juels 2006).

Despite the description of UT benefits and potential UT applications in the literature, organizations are still striving to determine whether and how they can benefit from UT. This seems to be attributable to a lack of understanding of the benefits of UT on a basic level. Fleisch and Tellkamp (2003) state that "so far there is only limited knowledge on the impact of UT on business processes and how applications based on these technologies can create value for companies". Similarly, Basole (2004) advocates the development of a framework suitable for identifying functional and process areas that can benefit from mobile technologies. The aim of this study is to identify a set of generic indicators for processes that can be improved by UT. The indicators can be used by companies to identify areas in which they may benefit from UT application.

The investigation draws on the theory of task-technology fit (TTF) which states that performance improvements can be achieved when the capabilities of a technology are aligned with the requirements arising from the tasks to be performed (Goodhue and Thompson 1995). In order to determine specific process characteristics that match with UT capabilities, a classification of UT capabilities was developed and a series of expert interviews were conducted to identify process improvements that could be achieved with specific UT functionalities. The cases reported by the experts were then analyzed to extract distinctive process characteristics that indicate whether a process is amenable to improvements by UT.

The remainder of this article is organized as follows: Sect. 2 presents the theoretical foundation, the research model, and a classification of capabilities of UT. The results of the expert interviews are documented in Sect. 3. The results of the analysis and comparison of the cases are presented in Chap. 4, resulting in a model explaining how UT usage can create value in business processes. Finally, a list of 
guiding questions is derived from the findings helping companies to identify the value creating potential of UT applications in their specific business process context.

\section{Theoretical foundations}

\subsection{Related work and research framework}

The benefits of UT in a business context have commonly been studied within a narrow scope. The applications and benefits of a single technology (e.g., RFID) are either assessed in a specific company, a certain industry, or in particular business processes (Boushka et al. 2002; Lampe et al. 2004). Consequently, the results of these studies can only be transferred to organizations that operate in the domain covered by the particular study. Some researchers have adopted a broader view examining the business impacts of UT on a conceptual level (Heijden and Valiente 2002; Yoo and Lyytinen 2003; Strassner and Schoch 2002). Strassner and Schoch (2003) found identification, monitoring, tracking, and notification to be the main capabilities of UT from which benefits can be accrued. However, the conceptual frameworks developed by this stream of research are not detailed enough to be directly applicable. For example, while Yoo and Lyytinen (2003) suggest that "complementary organizational assets and practices" combined with UT usage affect business process performance, no specific assets and practices that actually complement UT usage are identified.

The TTF, which was first proposed on the level of the individual IT users (Goodhue and Thompson 1995), while Zigurs and Buckland (1998) applied it on the organizational level, is a theory that has been applied to study the benefits of technology in organizations. A TTF is created if the capabilities of a technology fit with the characteristics of a task to be performed, which is a precondition for achieving performance improvements. We thus believe the TTF to be a suitable framework for better understanding the value-creation by UT in business processes. This is in line with a number of research studies that used TTF to explain the benefits of mobile technology usage. For example, Gebauer and Shaw (2004) used the TTF for explaining the benefits of mobile computing applications, which share similarities with UT applications such as the real-time aspect. Gebauer and Tang (2008) empirically confirmed the TTF for mobile applications. Moreover, a fit profile of mobile technology and managerial tasks was developed by Gebauer et al. (2006). Andersson and Lindgren (2005) matched task characteristics to capabilities of UT. Laubacher et al. (2005) attributed the benefits of RFID technology to improvements in specific families of activities and argue that looking for these activities enables companies to discover opportunities for value-creation.

We propose the following research framework for deriving characteristics of business processes that can be improved with UT (Fig. 1). In order to improve business process performance, a fit between the functionality of UT and the characteristics of the tasks to be performed in a company must exist. Relevant task characteristics are reflected by problems surfacing during process execution today, 


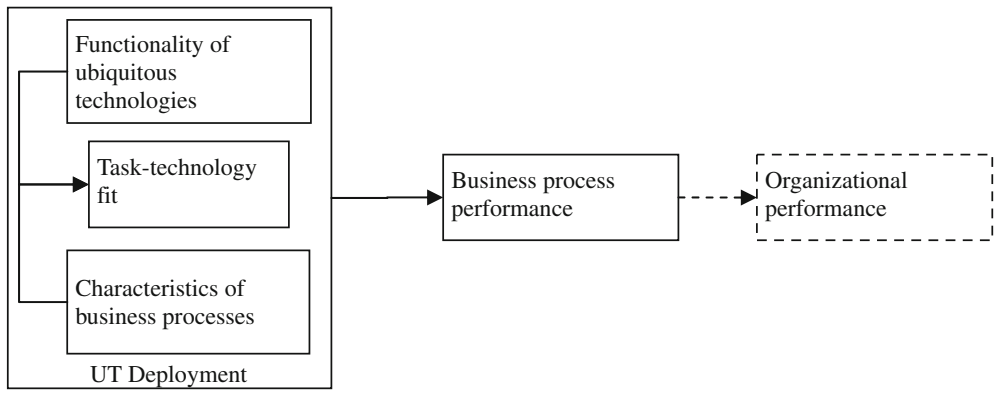

Fig. 1 Research framework

when no or only conventional IS support is available. The capabilities of UT allow to eliminate inefficiencies and to overcome some of these problems. It is important to note, however, that it is not the fit per se that generates positive business impacts, but rather the deployment of UT that fits with business process characteristics (see the box around the three boxes). Assuming that there are no interdependencies between the processes, aggregating performance gains over all processes in a company will result in an increased organizational performance.

\subsection{The functionality of ubiquitous technologies}

The functionality of UT has been derived from literature on instances of UT, such as sensor networks, RFID, and automatic identification systems. Any smart item must at least have the ability to store data and to communicate, but it can be more powerful. The functionality of an individual smart item does not depend on a specific technology such as RFID, but on the hardware components it is equipped with. Its classification is summarized in Table 1 and explained in the following.

\subsubsection{Data storage}

Traditionally, data about items associated with a company is stored in IS and there is no information flow between items and backend systems. In contrast, UT enable data storage directly on an item and subsequent data exchange with backend systems. Objects can carry data with them and make the data available wherever they are. For example, each item can store a serial number, which allows to uniquely identify individual items. The electronic product code (EPC) is a standard numbering scheme that was designed for this purpose (Brock and Cummins 2003). As a consequence, applications can be designed in which items are processed individually. This represents a paradigm change compared to today's business applications, in which objects are identified on product type rather than individual item level. However, UT applications do not generally require items to be uniquely identified. For example, in case of sensor networks, the focus is on data centric applications, in which the data reported is more important than the identity of items (Estrin et al. 1999). For example, multiple sensor nodes might record and report the 
Table 1 Classification of smart item functionality

\begin{tabular}{ll}
\hline Data storage about & Identity \\
& Item information \\
& Relationships \\
Data capturing of & Location \\
& Time \\
& Sensor measurements \\
& Presence of items \\
Data processing & Aggregate data \\
& Recognize events \\
Communication & Adjust state or behaviour \\
& Notify (Push) \\
Performing actions & Answer queries (Pull) \\
& Operate and control \\
& Generate output
\end{tabular}

temperature in a room, but the individual sensor nodes have no separate identity, as only the overall temperature is of interest. Besides identifiers, objects can store attributes like weight, colour or item manufacturer. If an item has sufficient memory capacity, larger files such as manuals or repair instructions can be stored. A relationship between objects can be established by storing the identifiers of corresponding objects.

\subsubsection{Data capturing}

The object related information described in the last paragraph cannot be created or captured by a smart item itself. This information needs to be sent to the item, e.g., from a business information system (BIS). There are, however, some data types that items can gather autonomously. An item can determine its relative or absolute position, e.g., by using GPS (Hightower and Boriello 2001). Capturing date and time information is a prerequisite for documenting the object history (Abowd and Mynatt 2000). Smart items may be equipped with physical, chemical, acoustic, or optical sensors, enabling them, e.g., to measure temperatures, monitor the composition of chemicals, take pictures of the environment or record sounds (Beigl et al. 2004). The communication device of a smart item may be used as a sensor for detecting nearby smart items, thus enabling contextawareness (Lyytinnen and Yoo 2003). The detection of other smart items in the neighborhood facilitates the collaborative execution of tasks (Estrin et al. 1999).

\subsubsection{Data processing}

Data offered by smart items shall be used to improve business processes that are supported by BIS. These systems are typically not able to handle large amounts of item-level real-time data and need to be shielded from the data flood generated by 
smart items (Bornhoevd et al. 2004). The data processing capabilities of smart items can be used to mitigate this problem by aggregating data as early as possible to a level that can be handled by BIS. As such, the data provided by smart items serves as a meaningful input for processes controlled by the back-end systems. For instance, instead of sending temperature values every second, an item can calculate and report average values hourly, while the back-end system triggers the dispatch of a service technician if the temperature exceeds a certain threshold. The processing capabilities of smart items also enable the identification of exceptions, e.g., in time series and events that need to be reacted to. Based on input about context and environmental conditions, smart items can adapt their state or behaviour. For instance, the expiry date of a product can be adjusted automatically to the observed storage conditions (Strassner and Schoch 2003).

\subsubsection{Communication}

Most smart items communicate wirelessly, contributing to a seamless integration in the real-world. Depending on the scenario, smart items may communicate with other items, mobile devices, BIS, or make use of networks. When communicating with BIS, two interaction modes can be distinguished: information pull (by an external system) and information push (by the smart item). Smart items may act primarily as data capturing and monitoring devices, answering queries issued by an external system about the state of the real world on demand. The process execution is controlled by the external system, while smart items act as "eyes and ears" of the system. On the other hand, smart items may execute and control parts of business processes autonomously, notifying external systems only in case they cannot handle events alone or require further input.

\subsubsection{Performing actions}

While some smart items are only able to capture and process information (passive items), active items can change the state of the real world and perform physical actions (Fleisch 2001). Embedded systems in particular are designed to operate and control real-world objects, e.g., to change the room temperature or to adjust the speed of production machines. Actuator components enable smart items to move, e.g., in response to changing environmental conditions. Smart items can also actively communicate with humans in their vicinity, e.g., by displaying information and issuing acoustic or optical warnings.

\subsection{Performance improvements}

To justify an investment in UT in a specific company, financial methods like calculating the net present value or the payback period have to be applied. The performance improvements in reference business processes cannot be quantified this way, as for quantifying costs and benefits, the context of implementation in an actual company needs to be known. For reference processes, the benefits need to be 
assessed on a more general level. According to Seibt et al. (1997), the benefits of IS can be attributed to one of the following five categories:

- effectiveness, e.g., improving output quality,

- efficiency, e.g., boosting productivity,

- cycle times, e.g., shortening cycle times or making them more predictable,

- flexibility, e.g., facilitating the change of processes, and

- customer satisfaction.

Moran et al. (2003) state that using UT can generate performance improvements in any of these categories. Besides improving existing processes, UT can also be employed to design new and innovative processes. With UT, new products and services can be designed, new forms of collaboration with business partners invented, and new business models created. Despite these potentially wider implications of UT usage on organizational performance, the scope of this article is limited to the impact of UT on existing business processes.

\section{Illustrative cases of ubiquitous computing applications}

\subsection{Data collection methodology}

A series of interviews with business process experts were conducted to identify processes which can be improved by UT and to understand why the capabilities of UT lead to performance improvements in a specific process. Expert interviews are a qualitative research method suitable for identifying patterns in UT applications (Andersson and Lindgren 2005). Compared to basing a study on cases reported in literature, expert interviews enable an in-depth understanding of the phenomenon (Gressgard and Stensaker 2006).

The 16 experts that participated in the study are employees of a provider of standardized business application software. They work as process architects, product managers, and experts for various industry specific applications. Hence they are familiar with a large number of companies and the respective business processes and can thus provide input on reference processes, which are executed in a large number of companies, in contrast to company specific processes. Furthermore, the experts have insights on the most pressing and common information needs and problems companies are facing today. This ensures that the process improvements identified answer real business needs and the process characteristics identified are relevant. As most ubiquitous computing applications in companies today are envisaged in the area of logistics, experts from other areas were targeted to ensure that the results of the study can be generalized.

A semi-structured interview guideline was applied. The participants were first asked about their field of expertise, the business processes they deal with, typical problems that companies executing these processes face and general industry pain points. Since the experts had no prior experience with UT, they were familiarized with the basic functionality of smart items described earlier. On the one hand, this helped to narrow down the set of reported problems to those that can be solved with 
the capabilities of UT. On the other hand, giving all experts the same introduction to UT ensured that the experts shared a similar level of knowledge about UT so that no potentials were missed due to a lack of understanding of the technology. The problems for which UT pose a potential solution and the surrounding processes were then analyzed in greater detail. Participants were asked to describe the process flow, people, objects, locations, and information systems (IS) involved. The problems surfacing in the process and the consequences of the problems were detailed further. In some cases, this close examination led to the discovery of further potential process improvements. The interview participants were eventually asked to estimate the benefits that companies could expect if the improved processes were implemented.

The method applied implies that the identified processes do not represent a complete or objective treatment of the phenomenon. It was not intended to discover cutting-edge application scenarios, but rather to identify potential improvements in reference processes from which many companies will benefit.

The interview results could be combined to nine application cases, as some of the interviewees described processes that were similar in structure but carried out in different industries. The cases are described in the next section, starting with a description of how the process is executed today, which problems exist, where and how UT can be employed to improve the process and how the process performance might be affected. Due to spatial limitations, not all cases can be presented in detail. Therefore, the main idea of nine application cases will be presented, while three illustrative cases will be described in greater detail. These cases have been selected for covering the range of problems that can be solved by UT as identified in this study. The three detailed cases are:

- Configuration control exemplified by aircraft maintenance,

- Asset tracking,

- Real-time production scheduling.

\subsection{Configuration control}

Configuration control is a software functionality used in aircraft maintenance that checks whether the configuration emerging after the replacement of an aircraft part is permitted. This check is necessary as different versions of components as well as components from different manufacturers may be incompatible with each other. Today, the configuration control is a step in a largely paper based process. When required, a service technician will be ordered to dismantle and replace a component of a plane. While on site, s/he records the IDs of both components on a sheet of paper, which s/he passes on to an engineer that typically resides in an office far off the maintenance site. The engineer enters the data in the BIS and triggers the configuration control. One problem is that the service technician will not pass on the maintenance record immediately, but will finish a certain amount of maintenance tasks before passing on the record. In the office, an engineer is not always present. It may take up to 2 days until the data is entered, which means that the configuration control is sometimes performed after a plane has long left the airport. A further 
problem in the maintenance process concerns the dismantled components. They are manually marked as "unserviceable", e.g., by putting a label on them. These labels may be overlooked or fall off, so that components that should be overhauled get mixed up with components already serviced. It thus might happen that an unserviceable component is installed in a plane.

The capabilities of smart items can help to overcome these problems. By utilizing their capability to uniquely identify nearby objects and to communicate with the backend systems, a solution can be designed in which the BIS is notified in real-time about any changes of the aircraft configuration. For instance, all critical parts of a plane could be equipped with RFID tags. If the service technicians are equipped with mobile devices, they can capture and immediately transmit the ID of the dismantled as well as of the new component to the backend system, which performs the configuration control and transmits the result back to the mobile device. In a more sophisticated scenario, the components of a plane could communicate with each other in order to determine autonomously whether the current configuration of the plane is permitted. Furthermore, the RFID tags on the components can store the state of a component (serviceable/unserviceable). If a technician tries to install a part marked as unserviceable in a plane, a warning can be issued.

When optimizing the process by using UT, the handling of information recorded on paper is avoided. This reduces labour costs. The process will run faster, as waiting times are eliminated. However, the most important benefit is the reduction of errors, as they may have severe consequences in aviation. For instance, an emergency landing that was caused by technical failures due to incompatible components is costly and damages the image of the carrier. Errors in safety critical domains can entail high costs and are likely to damage the image of a company. Companies operating in these domains will thus generally be willing to invest in innovative technologies if they promise to boost safety.

\subsection{Asset tracking}

Information about the assets a company owns and their location is stored in an asset management system. The term "asset" refers to all kinds of technical objects, ranging from laptops and forklifts to entire production plants. Locations are represented as hierarchical structures, that contain, e.g., buildings, floors and rooms. Today, the location of an asset is entered manually or captured by scanning a barcode that is attached to the doors of a building. It is often forgotten to initially assign a location to an asset or to update the location once an asset is moved. Manual data entry results in wrong location assignments. The consequences of incorrect asset data include inaccurate inventory levels, people spending much time looking for assets, difficulties in assigning asset costs to departments, and the inability to maintain and service assets as they are not found at the expected location. An additional problem with mobile assets such as laptops or beamers is that they might be stolen. In this case, the asset and location will be stored in the system, but no longer be present in the company.

With UT, an infrastructure can be created that allows at least in principle to identify and localize each asset at any time and to check for its presence. For that 
purpose, it is necessary to turn the assets into smart items, e.g., by putting RFID tags on them. RFID readers can then be installed in the doors, rooms or corridors of buildings. By propagating asset data to the backend systems, e.g., every time an asset is moved, it can be ensured that the correct asset location is maintained in the database. Furthermore, stocktaking can be automated, e.g., by querying all readers for all objects in their read range. This could replace or facilitate the stocktaking process carried out today. Once assets have been turned into smart items and are tracked, this can be used for theft prevention. For instance, the exits of a building can check whether assets passing through them are permitted to leave the building and issue an alarm if necessary.

Optimizing asset management will raise employee productivity and cut down labour costs since people spend less time looking for assets, manually assigning assets to locations, revising wrong data in the system, and stocktaking. An automated stocktaking solution will furthermore avoid the halt of operations often associated with stocktaking today. The decline in asset theft will lower the expenses for replacing stolen assets. Making the number and location of assets more transparent will prevent the unnecessary replacement of undetected or hidden assets which sometimes happens today.

\subsection{Real-time production scheduling}

An enterprise manufacturing solution determines the optimum production schedule for a given set of orders, while taking into account multiple constraints, e.g., the number of available production machines or the amount of preliminary products. When the optimum schedule for a predetermined time period has been established, the schedule is fixed and production starts. This makes the production process quite inflexible and often suboptimal, as no data about the actual state of the shop floor is incorporated and used to optimize the schedule once production has started. Besides the inability of many enterprise systems to handle large amounts of real-time data, the lack of available data poses a problem in itself. In some companies, no information about the location and amount of work in progress cycling on the shop floor, the state of production machines, or the availability of preliminary products is reported back to the manufacturing system, where it could be used to update the production schedule. Thus, if an unexpected event happens, such as a breakdown of a machine, the schedule cannot be immediately adjusted. Although some companies use automatic or manual production data acquisition systems, this is no real improvement as the data captured is only used retrospectively for generating reports about finished processes.

UT can be employed to constantly monitor the state and location of all objects on the shop floor (including preliminary and finished products, machines, transport devices, containers and people), to aggregate the huge amounts of data before feeding them to the manufacturing system, and to identify and report exceptions. The condition and status of machines and products can be monitored with sensors and the values can be fed back to the manufacturing system. If rules specifying valid production processes are violated, the backend system can be notified. 
Compared to the data captured by today's production data acquisition systems, the level of detail and comprehensiveness of the data can be extended, e.g., by capturing the processing times and conditions of individual objects. The detailed information provided by the smart items can then be used by the manufacturing systems to optimize the production schedule based on the actual situation.

As manual or semi-automated production data acquisition becomes obsolete with the introduction of ubiquitous computing applications, labour costs will be reduced. Even larger savings can be expected from optimized production schedules. Significant increases in productivity can be expected when better schedules are generated. Companies will be able to better predict the completion times of their products and to issue more reliable delivery dates. Moreover, as the manufacturing conditions of individual products can be tracked, companies will be able to prove the compliance to regulations and the absence of errors during production, which may help to reduce penalties.

\subsection{Further applications}

Based on the expert interviews, six more application cases could be identified, which are briefly sketched here:

- Automatic update of the installed base: UT can be employed to ensure that information about the configuration and structure of multi-component objects is kept up-to-date in the BIS. The individual components of objects can be tagged and version information can be stored on the tags. Thus, the handling of configuration information during manufacturing, maintenance and when objects are transferred to a third party are improved.

- Condition based maintenance: In this scenario, information about the condition of an asset is captured by sensors and its daily operations are monitored. This information is used to predict when the next maintenance needs to take place. Preventive maintenance helps to reduce the downtime of assets because they are serviced before they can break down and thus increases productivity.

- Mobile asset maintenance: UT allow to improve the mandatory maintenance of fire vents and other safety critical components, particularly when the correct performing of maintenance must be proven to authorities because of legal regulations. RFID tags are attached to the components and the service technicians are equipped with mobile devices. When a technician approaches a component, the tag and the mobile device communicate with each other which ensures that the service is performed correctly. The former error-prone paperbased process is replaced and the storage of large amounts of maintenance documents can be omitted.

- Optimizing the selection of materials that are consumed during manufacturing: In this scenario, additional information about the state of the preliminary products, e.g., their temperature, viscosity, expiry date or their position in the warehouse is used to optimize the initial production schedule calculated by the system and to reduce waste. 
- Tool management: UT can help to predict when tools used on the shop floor, such as extrusion rams, need to be replaced due to deterioration. Tools consist of various components with varying life spans. The life span of individual components may depend on certain attributes of the handled product (e.g., soft/ hard metal). Tagging of the components of the tool and the processed objects can help to predict when a tool needs replacement because the maximum life span of one of its components is reached.

- Picking and final checking in warehouses: It needs to be checked whether goods picked in a warehouse match with the orders. With the help of UT, the identity and type of objects can be queried and transmitted electronically. This capability can be used to facilitate the picking process and to check whether the content of picking boxes or containers is complete and correct.

\section{Data analysis and implications}

\subsection{Approach}

To identify the characteristics that make a process amenable to improvements by UT, the cases gathered from the interviews were first split up into their building blocks, each representing a potential UT application. For example, the case "configuration control" (Sect. 3.2), includes (1) checking the configuration of an asset after components have been changed as well as (2) proper handling of dismantled components. For each building block, the problems in today's processes that can be solved by UT were extracted. These problems reflect the process characteristics that indicate a beneficial UT application. For instance, problems in performing configuration control are due to manual data entry resulting in delays and errors, a delayed reaction to configuration changes due to a paper based process and the requirement to have a specifically trained technician performing the task who is not always available and lastly, the nature of the task being to check the consistency of particular item combinations, i.e., the types of components being installed together. Table 2 gives an overview of the UT applications reported by the experts, the problems that can be solved by UT in the processes, and the combination of UT capabilities that can be employed to overcome the problem. The numbers behind the problem types refer to the description of indicators in Sect. 4.2.

By grouping the applications by problem type and comparing the UT functionality needed to overcome the problem, it can be inferred why UT are suited to solve a certain problem type and what exactly causes the fit between UT and a particular process characteristic.

The matching of UT capabilities and problem types is illustrated in Table 3 for the problem type "data is needed on site". This example covers two applications: A service technician performing condition based maintenance and a component change. In both cases, the service technician needs up-to-date and correct information about the object s/he has to service (ID, attributes, aggregated sensor measurements), which is not directly available to her/him today. In the improved 


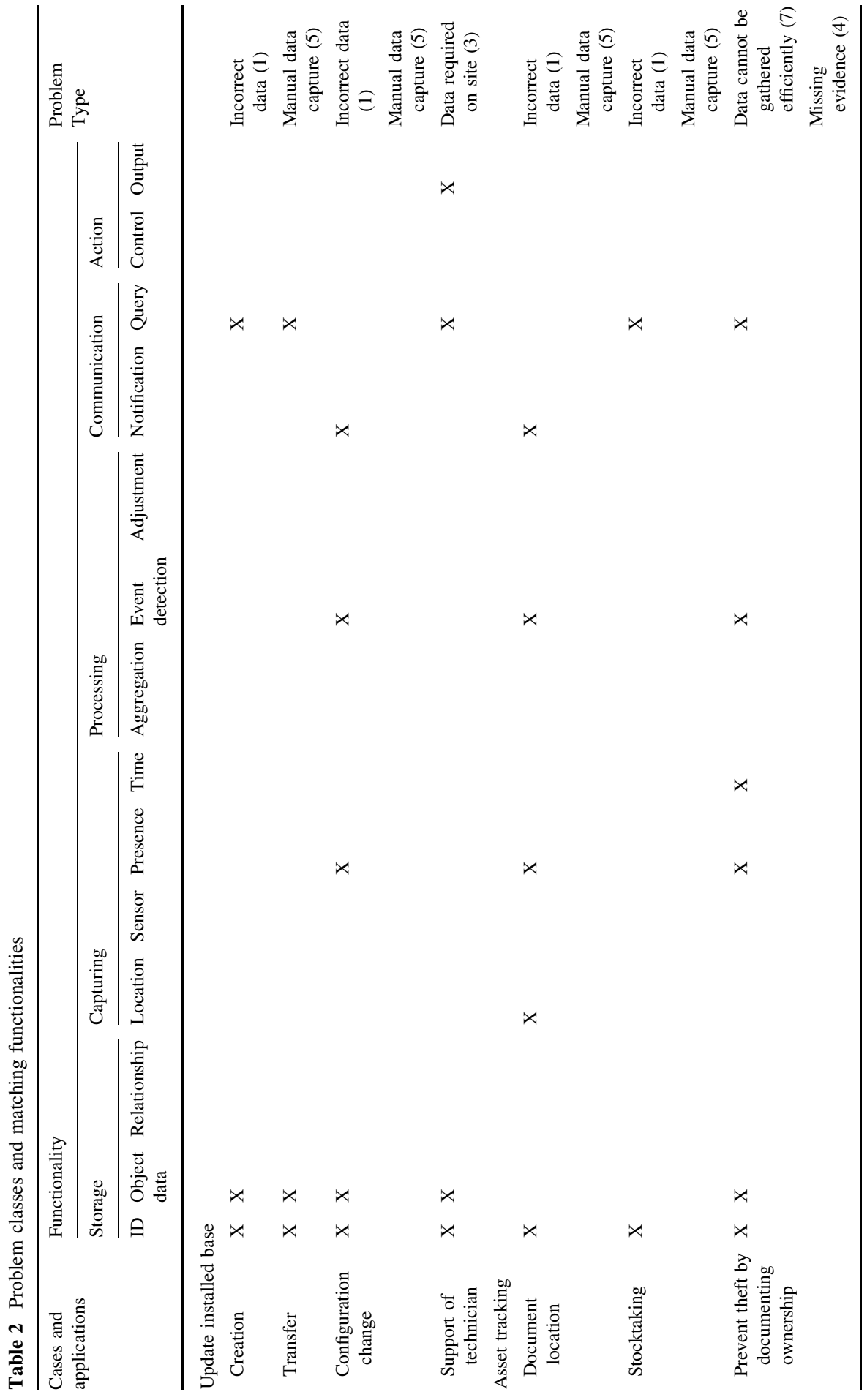




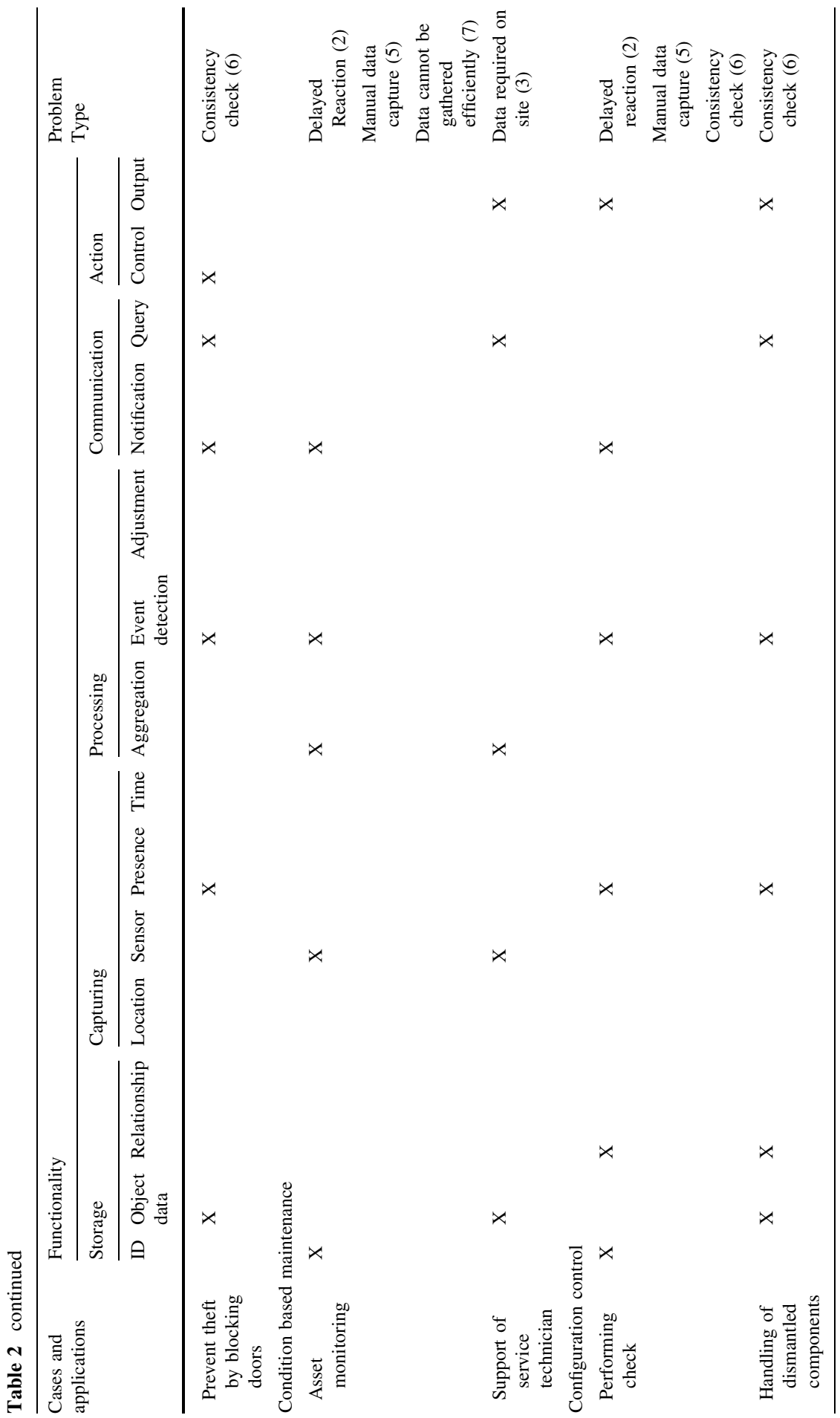




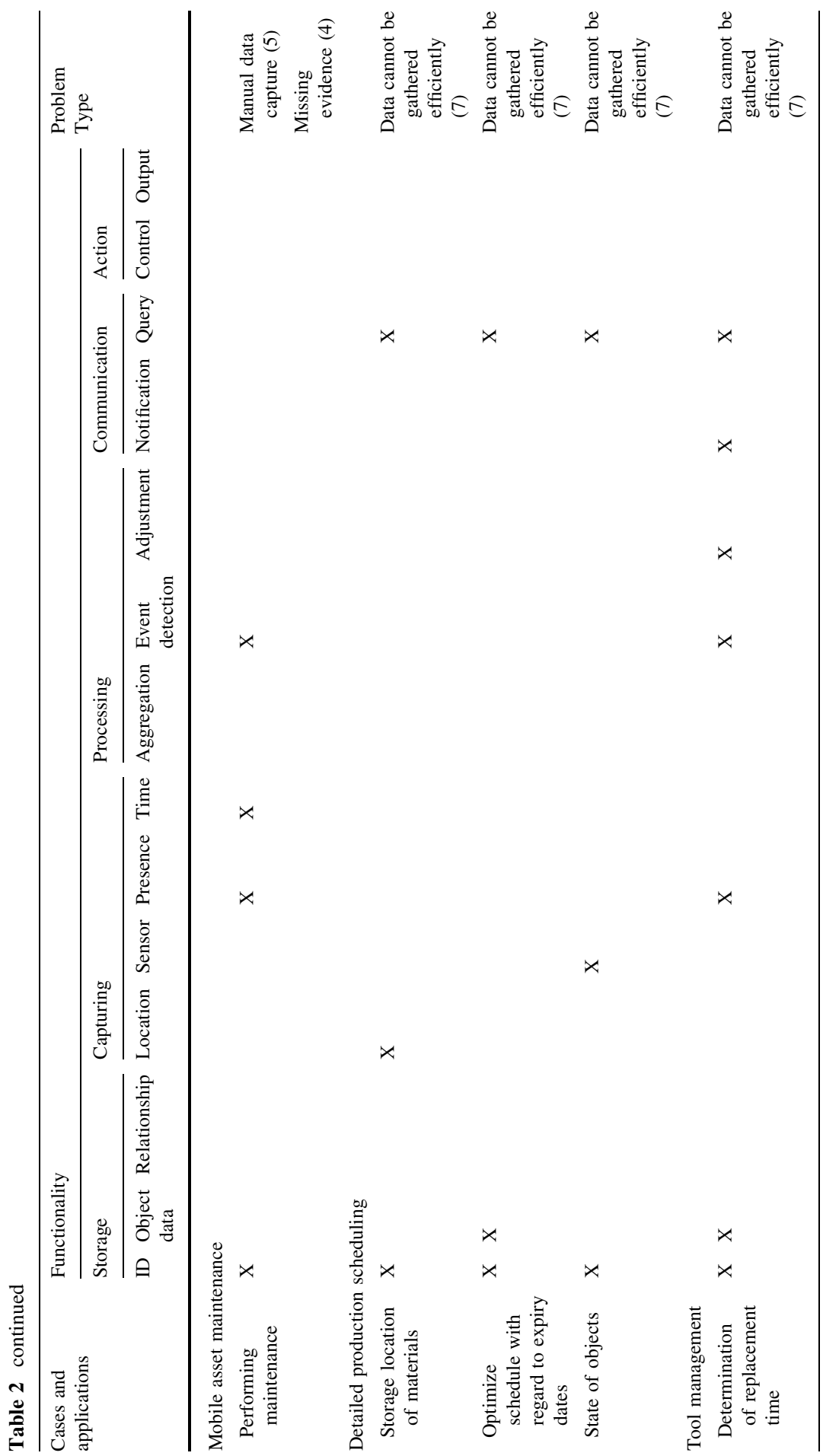




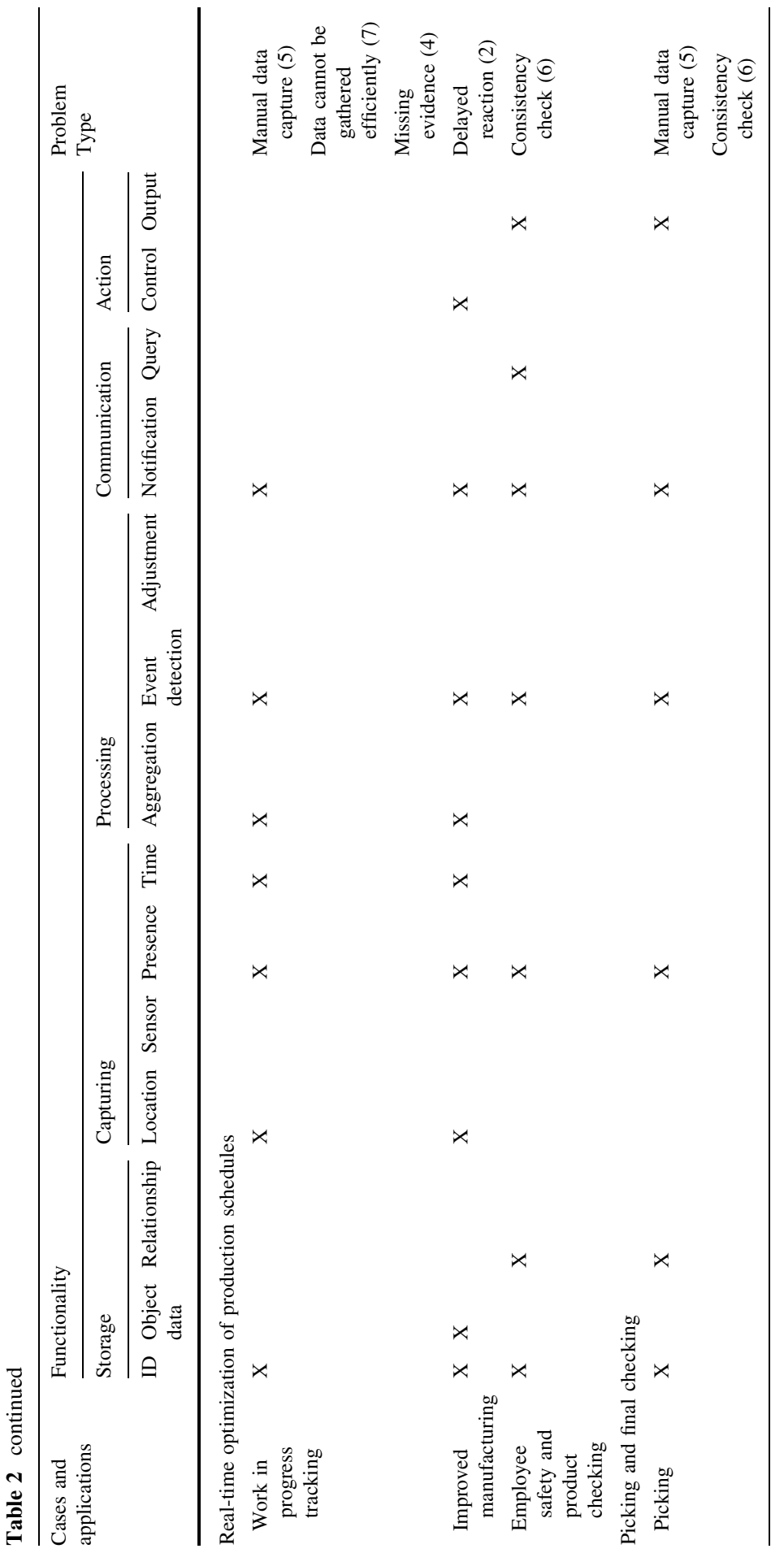




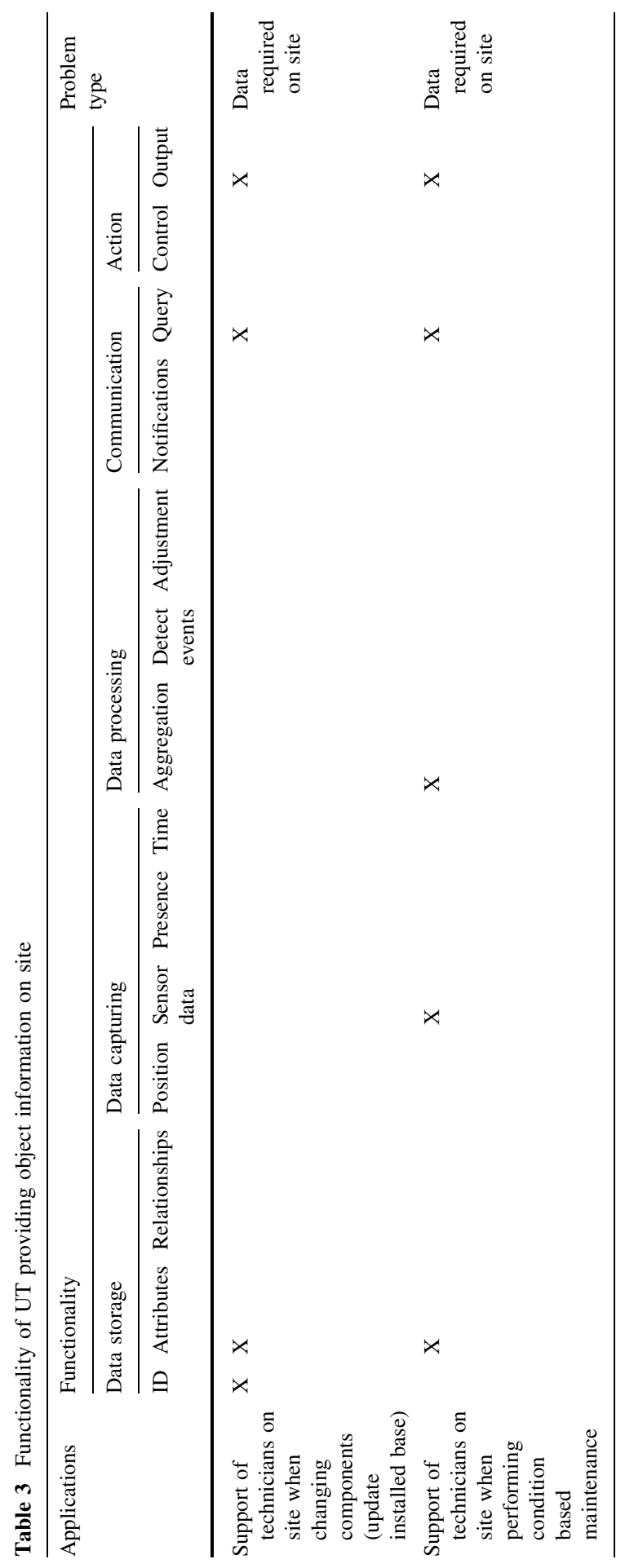


process, s/he will be able to query the object directly for relevant information, and the data will be sent to her/his mobile device or displayed on the object itself. The capabilities of UT that help to overcome the problem of a lack of data at the location where it is required are: the storage of useful data on the object (data storage capability) and the ability of smart items to report that information when queried (capability to answer queries). In case sensor data is relevant for the process to be performed on site - as in the case of condition based maintenance-the capturing and aggregating of the data provides useful functionality (capability to capture sensor data, capability to aggregate data). Whenever up-to-date information (whether captured or static) about objects is needed in the real-world and no access to IS is available, the functionality of UT is thus suited to overcome this problem and increase process performance.

The results of the analysis across all applications and problem classes are presented in the next section.

\subsection{Indicators of improved process performance with UT}

The following seven indicators of potential process improvements by using UT could be identified:

1. The first type of problem that may be solved with UT is the storage of incorrect information about the state of the real world in IS. This includes object attributes such as temperature, location or configuration, but also aggregated values such as inventory levels. The reason for a discrepancy between the actual state of the world and the state represented in the IS can be attributed to the media break between the real world and the virtual world (Fleisch 2001). As the state of the real world is not automatically transmitted to the IS, a manual data entry is necessary which may entail errors and omissions. Problems surface when the false data is used to take decisions, which are in consequence wrong or suboptimal. The data utilized in process execution is required to be correct, otherwise process performance will decline. There are two ways in which UT can contribute to overcome this problem. First, the backend system can query the smart items about the state of the real world before taking a decision. Second, smart items can constantly monitor the state of the real world and, as soon as relevant changes occur, notify the central systems. This ensures that the decisions taken are based on correct input values.

2. The next problem that can be tackled by using UT is a delayed reaction to events in the real world. For instance, a delayed reaction was reported for plane component changes. Other examples include broken down production machines or tools that are not replaced immediately, because it takes too long to detect the error and to inform the responsible person about the event. Generally, delays may be caused by waiting times in paper-based processes or by a missing systematic monitoring of the real world, e.g., when people discover urgent problems by mere chance. In the latter case, the ability of smart items to notify backend systems about critical events in real-time is of special importance. Beyond that, it is also possible to design processes that are no longer controlled 
by backend systems. Smart items can detect important events and react to them autonomously and immediately, e.g., by issuing a warning when the rules specifying the configuration of an airplane are violated.

3. UT can be employed when data is required on site, e.g., at the place where an object resides. This may be the case when no IS is available, e.g., in warehouses or hangars, but also when access rights for retrieving information are missing, e.g., if objects are transferred to another company. Smart items are well suited for solving this kind of problem because they carry information about themselves with them, all the time and everywhere they go, and this information can be transmitted to a requester. Thus, smart items can meet all local information needs. The immediate availability of information also facilitates the exchange of products and associated data between organizations.

4. If companies need to (e.g., because they must comply with regulations) give evidence about the lifecycle of objects and achieve accountability, they can profit from UT. Compared to today's methods of monitoring the lifecycle of objects, which often involve heavy paperwork, UT offer a possibility to make monitoring much more reliable. As the physical and the information processing component of a smart item are continuously connected, the entire lifecycle of an object can be observed and recorded by the object itself, e.g., changes in state, actions taken, or objects the item interacted with. This allows companies to prove that assets were serviced regularly, or that the production process was carried out according to specifications.

5. In most of the examined processes, an inefficient manual or semi-automated (using barcodes) data entry and data capturing was performed. Process steps carried out manually may not only cause errors and delays, but they also produce labour costs. Many paper-based and manual processes can be automated using UT, as long as the process deals with real-world objects. Smart items can capture the data needed and send all relevant information automatically to the target systems. Thus, manual data gathering and data entry are avoided.

6. UT can enhance scenarios in which the state of the real world regarding objects and their attributes is not checked adequately. A correct state of the real world may depend on the presence or absence of certain objects, on the completeness of a set of objects, or on a correct combination of object attributes. Examples include the check whether shipments are complete and their composition is correct, the check whether a worker has the required training to operate a machine, whether a component is serviceable or whether an asset is authorized to leave a building. Today, these checks are either performed manually, sporadic, or not at all. UT not only allow to perform these checks automatically, but they can also ensure that the rules pertaining to a correct world state are observed continuously. In case the rules are violated, a warning can be issued or actions triggered that aim at restoring a correct state.

7. Quite often, companies are aware of the data input needed to improve the decision quality, but with the available technologies, this information about the state of the real world cannot be gathered efficiently enough. Accordingly, they rely on heuristics or estimates to compensate for the missing data. Measures for 
gathering the data can be envisioned in many cases, but implementing them would be too costly compared to the benefits of an improved decision quality. As smart items are cheap and efficient data gathering devices, they can be used to supply IS with decision improving data at little cost. For example, UT enable monitoring of individual items instead of product aggregations, more attribute values can be kept track of, and, in general, data about more objects can be stored in IS. For example, while today only few expensive or mission critical objects are connected to the backend systems for monitoring their condition and scheduling preventive maintenance, many more objects can be hooked up to the backend system when UT are used to capture and aggregate data before transmitting it.

\subsection{Moderating factors leveraging performance gains from UT}

In addition to the problem types, the comparison of UT applications revealed another pattern. Four moderating factors could be identified that leverage the extent to which performance gains can be expected when UT is used to improve processes: a high object price, safety critical operations, the need to comply with regulations and high costs of asset downtime.

1. UT can help to prevent objects from being damaged, stolen or getting lost because they enable a close monitoring, tracking and checking of objects. The higher the object price is, the higher are the costs for replacing an asset and the bigger is the incentive for companies to invest in UT to avoid these costs.

2. When dealing with risky or dangerous objects or processes, the costs of errors are potentially high. Furthermore, when performing safety critical operations, people might get injured and an incident may have severe consequences on the image of a company. As UT help to prevent errors by replacing human labour and by checking actions performed by humans, companies conducting safety critical operations will be more likely to invest in promising technologies.

3. UT can help companies in various ways to ensure and to prove that they comply with regulations that are determined e.g., by public authorities. Today, many companies spend an enormous effort for complying with regulations with traditional means. UT offer in many cases a cheaper and more reliable alternative.

4. UT can be employed to maximize the uptime of assets and to increase their productivity. This can be achieved by enabling preventive maintenance, by optimizing production schedules or by quickly restoring normal operations in case of unexpected events. The higher the costs of asset downtime, the more willing will companies be to invest in UT.

Figure 2 summarizes these findings by integrating the identified factors in the research framework. The model illustrates how the functionality of UT in conjunction with a specific set of problem types (i.e., process indicators) creates a $\mathrm{TTF}$, which in turn affects the process performance and organizational performance. 


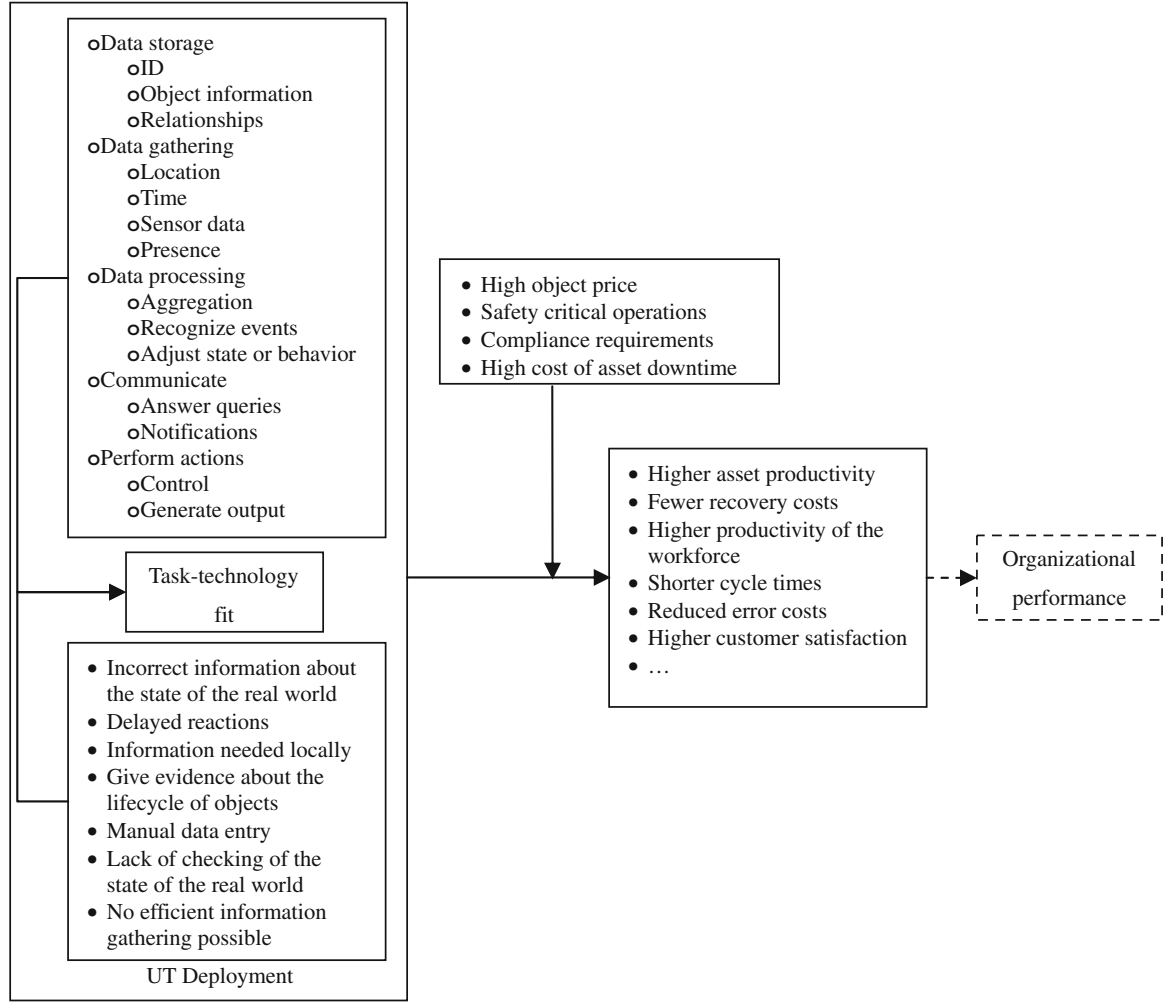

Fig. 2 Model of value creation by UT

The initial model was extended to account for additional factors influencing the extent of performance improvements.

\subsection{Practical application}

The results of this study can be applied to help companies identify value-creating ubiquitous computing applications in their specific context. The problems identified are indicators of potential process optimizations. Thus, the following questionnaire reflects the seven task characteristics as depicted in the lower left-hand box of Fig. 2 as well as the moderating factors. It can be employed to identify areas in which UT usage can be beneficial. Interviews with company representatives based on this questionnaire may be used to develop initial ideas for UT usage. For the purpose of matching specific task characteristics with UT features, Table 2 may be used as a baseline. Once these processes have been identified in the first step, it remains to be assessed in greater detail whether the envisioned process changes are technically and economically feasible. In the second step, the potential impacts in the context of the company thus need to be identified, the costs and benefits of the envisioned solution need to be quantified and the risks and privacy implications need to be 
weighed in. However, the identification of processes which might be improved by UT needs to precede this step. Questions to be asked for this purpose include:

1. In which cases is the state of the real world not reflected correctly in the IS? In which processes does this lead to wrong decisions and with which consequences?

2. In which processes are reactions to events in the real world delayed? For which decisions do you need real-time data?

3. In which processes have employees no access to IS or work in an offline scenario, but need object information?

4. Which measures do you employ to give evidence about the lifecycle of objects? In which situations do you need to give hard evidence?

5. In which processes is object information entered, captured or transmitted manually? Where do media breaks occur? Which processes dealing with objects are paper-based?

6. In which processes is the completeness, composition, absence or presence of real world objects checked? In which cases are documents accompanying an object checked? In which processes is the process flow determined by object attributes?

7. In which processes can the decision quality be improved when more and better information about the state of the real world is available? In which cases are objects not monitored because this is too costly?

(a) In which processes and for which objects emerge high replacement costs due to loss or damage?

(b) In which processes must the safety of operations be guaranteed? In which cases may errors have severe consequences for the safety and health of people?

(c) For which objects and object related processes is compliance required? Which object information has to be documented due to legal regulations?

(d) For which objects is an unproductive period extremely costly? In which processes do objects have to be continuously available and ready to operate?

\section{Conclusion and outlook}

A set of indicators for value creating ubiquitous computing applications in a business environment have been identified. These indicators represent process (i.e., task) characteristics that hint at problems that can be solved by employing UT. Moreover, moderating factors were identified that leverage the extent to which benefits can be achieved from using UT in support of specific business processes. The process indicators were identified by applying the theory of TTF to ubiquitous computing in a business context. The TTF can thus be seen as kernel theory that was applied with the objective to support the conceptual development of UT solutions that support particular business processes (Walls et al. 1992). By illustrating the relationships between particular task characteristics of business processes and particular UT functionalities (see Table 2) as well as the impact of their combination on business process performance, a first step towards a design theory 
of UT-supported business process has been conducted (Walls et al. 1992). The identified task characteristics represent a set of typical problems existing in today's business processes. These problem domains where operationalized in form of a questionnaire that helps practitioners to identify those business processes in which particular UT can yield the most positive impacts on business process performance.

It should be kept in mind, however, that while these indicators are quite comprehensive, they are neither assumed to be complete nor entirely disjunctive. By examining further case studies, the identified problem types should be verified and additional problem classes be determined. The proposed questionnaire for identifying value-creating applications should be put to practical use in companies for identifying new UT applications. Methods for assessing and quantifying the impact of UT once potential applications have been identified are not readily available and could be a target for future research.

\section{References}

Abowd GD, Mynatt ED (2000) Charting past, present and future research in ubiquitous computing. ACM Trans Comput Hum Interact 7(1):29-58

Andersson M, Lindgren R (2005) The mobile-stationary divide in ubiquitous computing environments: lessons from the transport industry. Inf Syst Manage 22(4):65-79

Basole RC (2004) The value and impact of mobile information and communication technologies. Proceedings of the 2004 IFAC symposium, Atlanta, GA

Beigl M, Krohn A, Zimmer T, Decker C (2004) Typical sensors needed in ubiquitous and pervasive computing. Proceedings of the first international workshop on networked sensing systems. http://www.teco.edu/ krohn/inss.pdf. Accessed 01 Jan 2009

Bornhoevd C, Lin T, Haller S, Schaper J (2004) Integrating automatic data acquisition with business processes-experiences with SAP's Auto-ID infrastructure. In: Nascimento M, Öszu M, Kossmann D, Miller R, Blakeley J, Schiefer K (eds) Proceedings of the 30th international conference on very large databases, pp 1182-1188

Boushka M, Ginsburg L, Haberstroh J, Haffey T, Richard J, Tobolski J (2002) Auto-ID on the move: the value of Auto-ID technology in freight transportation. Auto-ID center white paper. http://www. autoidlabs.org/uploads/media/ACN-AUTOID-BC-003.pdf. Accessed 01 Jan 2009

Brock D, Cummins C (2003) EPC tag data specification. Auto-ID center white paper. http://www. autoidlabs.org/uploads/media/MIT-AUTOID-WH025.pdf. Accessed 01 Jan 2008

Estrin D, Govindan R, Heidemann J, Kumar S (1999) Next century challenges: scalable coordination in sensor networks. In: Proceedings of the 5th annual ACM/IEEE international conference on mobile computing and networking, pp 263-270

Fleisch E (2001) Business perspectives on ubiquitous computing. M-Lab working report Nr. 4, 2001

Fleisch E, Tellkamp C (2003) The challenge of identifying value-creating ubiquitous computing applications, Workshop on ubiquitous commerce, UbiComp 2003

Gebauer J, Shaw M (2004) Success factors and impacts of mobile business applications: results from a mobile e-procurement study. Int J Electron Commer 8(3):19-41

Gebauer J, Tang Y (2008) Applying the theory of task-technology fit to mobile technology: the role of user mobility. Int J Mob Commun 6(3):321-344

Gebauer J, Shaw M, Michael J, Gribbins ML (2006) Task-technology fit for mobile information systems, working paper, University of Illinois School for Business, No 06-0107. http://www.business.uiuc. edu/Working_Papers/papers/06-0107.pdf, 2006. Accessed 01 Jan 2009

Goodhue D, Thompson R (1995) Task-technology fit and individual performance. MIS Q 19(2):213-236

Gressgard L, Stensaker I (2006) The mobile service industry: strategic challenges and future business models. Int J Mob Commun 5(4):509-531 
Heijden H, Valiente P (2002) Mobile business processes: cases from Sweden and the Netherlands. Stockholm school of economics working paper series in business administration 14

Hightower J, Boriello G (2001) A survey and taxonomy of location systems for ubiquitous computing. IEEE Comput 34(8):57-66

Inaba T (2008) EPC system for a safe \& secure supply chain and how it is applied. In: Cole P, Ranasinghe D (eds) Networked RFID systems and lightweight cryptography: raising barriers to product counterfeiting. Springer, Berlin, pp 191-210

Juels A (2006) RFID security and privacy: a research survey. IEEE J Sel Areas Commun 24:381-394

Lampe M, Strassner M, Fleisch E (2004) An ubiquitous computing environment for aircraft maintenance. In: ACM symposium on applied computing, Nicosia, Cyprus, pp 1586-1592

Laubacher R, Malone TW, Kothari SP (2005) What is RFID worth to your company? Measuring performance at the activity level. MIT center for coordination science working paper, 2005

Lee H, Özer O (2007) Unlocking the value of RFID. Prod Oper Manage 16(1):40-64

Lyytinen K, Yoo Y (2002) Issues and challenges in ubiquitous computing. Commun ACM 45(12):63-65

Mattern F (2005) Ubiquitous computing: scenarios for an informatized world. In: Zerdick A, Picot A, Schrape K et al (eds) E-merging media: communication and the media economy of the future. Springer, Berlin, pp 145-164

Moran H, McFarlane D, Milne T (2003) Use case approach for determining the impact of Auto-ID implementations on business information systems. Auto-ID center white paper. http://www. autoidlabs.org/uploads/media/CAM-AUTOID-WH020.pdf. Accessed 01 Jan 2009

Satyanarayanan M (2002) A catalyst for mobile and ubiquitous computing. IEEE Pervasive Comput Mag $1(1): 2-5$

Seibt D, Bielli P, Bjorn-Andresen N et al (1997) CEBUSNET consolidation framework. Working paper No.1/1997, chair of information systems and information managements, University of Cologne

Strassner M, Schoch T (2002) Today's impact of ubiquitous computing on business processes. In: Mattern F, Naghshineh M (eds) Short paper proceedings of the international conference on pervasive computing 2002, pp 62-74

Walls J, Widmeyer G, Sawy O (1992) Building an information system design theory for vigilant EIS. Inf Syst Res 3(1):36-59

Wamba S, Ygal B (2008) Understanding the impact of emerging technologies on process optimization: the case of RFID technology. 13th Asian-Pacific decision sciences conference, Brisbane, Queensland, Australia, 25 July 2008

Weiser M (1993) Some computer science issues in ubiquitous computing. Commun ACM 36(7):75-84

Yoo Y, Lyytinen K (2003) Measuring the consequences of ubiquitous computing in networked organizations. Sprouts: working papers on information environments, systems and organizations, 3(3)

Zigurs I, Buckland B (1998) A theory of task-technology fit and group support systems effectiveness. MIS Q 22(2):313-334 